\title{
GCU
}

Glasgow Caledonian

University

University for the Common Good

\section{Dimensions of personal meaning from engagement in occupations: a metasynthesis}

Roberts, Anne E. K.; Bannigan, Katrina

Published in:

Canadian Journal of Occupational Therapy

DOI:

$10.1177 / 0008417418820358$

Publication date:

2018

Document Version

Author accepted manuscript

Link to publication in ResearchOnline

Citation for published version (Harvard):

Roberts, AEK \& Bannigan, K 2018, 'Dimensions of personal meaning from engagement in occupations: a metasynthesis', Canadian Journal of Occupational Therapy, vol. 85, no. 5, pp. 386-396.

https://doi.org/10.1177/0008417418820358

\section{General rights}

Copyright and moral rights for the publications made accessible in the public portal are retained by the authors and/or other copyright owners and it is a condition of accessing publications that users recognise and abide by the legal requirements associated with these rights.

Take down policy

If you believe that this document breaches copyright please view our takedown policy at https://edshare.gcu.ac.uk/id/eprint/5179 for details of how to contact us. 
Dimensions of personal meaning from engagement in occupations: A meta-synthesis.

\begin{abstract}
Background. Occupational scientists have offered various theoretical propositions regarding the dimensions of meaning derived from engagement in occupations and empirical studies have explored the personal meaning of engaging in a wide range of occupations. Purpose. The study aimed to distil the dimensions of personal meaning from existing research, with a view to informing theory in this area. Method. A qualitative metasynthesis of research studies was conducted, focusing on research reporting individuals' accounts of the personal meaning of an occupation to them. Twenty papers met the search criteria and were reviewed, rated for quality, analysed and synthesised following a process of meta-aggregation. Findings. There were four common dimensions of personal meaning: a sense of fulfilment; a sense of restoration; social, cultural and intergenerational connection and identity shaping.

Implications. This review provides occupational therapists with sound evidence of the link between engagement in a meaningful occupation and a sense of personal and social wellbeing.
\end{abstract}

\title{
Introduction
}

\section{Aim and Purpose}

Within occupational science it is acknowledged that humans are occupational beings (Yerxa et al 1989). It is accepted that the occupations we choose to do hold meaning (Polatajko et al., 2013a) and within occupational science and occupational therapy there is a belief that being engaged in a meaningful occupation is related to health and wellbeing (Wilcox, 1998). It is less well understood what the dimensions of meaningful occupation are, although there have been many theoretical attempts to describe these (Pierce 2001a; Hammel 2004, 2009a, 2009b; Jonsson 2008). The personal meaning of a wide range of different occupations has been researched and reported, for example; creative occupations that have a tangible end-product like cake decorating (Sheerer, Cahill, Kirby, Lane, 2004); 
occupations that create a transient art form like performing in a band (Roberts \& Farrugia, 2013); active sporting occupations like dragon boat racing (Unruh and Elvin 2004). Although there is some variation in meanings depending on the occupation, the individual and the context, anecdotally there also seems to be some similarity in the meanings that people ascribe to these varied occupations. It would be helpful for occupational therapists and professionals promoting a healthy lifestyle to understand what specific meanings an occupation chosen by an individual has for that individual, as this can be used to tailor intervention to enhance specific benefits to health and wellbeing. Furthermore, it is helpful to know if some occupations provide more opportunities for meaningfulness than others, for example whether creative occupations provide more, or different types of meaningfulness and thus therapeutic opportunities compared to say, active sporting occupations. This information can be provided by a synthesis of the extant body of research on the personal meaning of engagement in a wide range of different pleasurable occupations. Synthesis of this information would also progress a theory of any common dimensions of meaning across occupations chosen by individuals for their intrinsic benefits. These empirically based dimensions can then be related to existing theory and confidently applied by occupational therapists in using occupations therapeutically to enhance or maintain health and wellbeing.

\section{Literature Review}

\section{Meaning of Occupations}

In this paper occupation is defined as "an activity or set of activities that is performed with some consistency and regularity that brings structure, and is given value and meaning by individuals and a culture" (Polatajko et al., 2013a, p. 19). Although occupations can be viewed as things people "need to, want to and are expected to do" (WFOT, 2015), in this paper the focus is on occupations that we want to do, that we have chosen to do because they are pleasurable and satisfying in and of themselves. Csikszentmihalyi (1992) called these 
"autotelic" occupations: "self-contained activity, one that is done not with the expectation of some future benefit, but simply because the doing itself is the reward" p. 67. The occupations we choose to do for their own sake have meaning to us and meaning is idiosyncratic (Polatjko et al., 2013b). Sometimes these occupations are leisure occupations, but this term does not adequately define cultural occupations that may be part of a person's life in a non-western context. Nor is leisure a uniform concept; occupations that are leisure for some people are work for others (Hammell, 2009a). For example, baking may be productive work for a mother or father feeding their family, or leisure to someone baking for the sheer pleasure of doing it. Furthermore, the categories of productivity and leisure used extensively in occupational therapy theory are not sufficiently linked to evidence and are used with inadequate justification in the literature (Hammell, 2009b).

Current theoretical guidance suggests that our choice of meaningful occupations is unique to us as individuals, defining us as a person, giving us a sense of identity (Hinojosa \& Kramer, 1997). These occupations usually occur in social, family and cultural contexts (Hasselkus, 2011) and these social aspects enhance their meaning. Meaning is defined here as "what we create for ourselves in our mind that explains experiences and, in turn, motivates us and spurs us on to create new experiences" (Polatajko et al., 2013b, p. 61).

\section{Dimensions of meaning}

In the occupational therapy and occupational science literature there has been discussion about the best way to describe the characteristics or dimensions of occupations. A typographical method of categorising occupations as self-care, productivity and leisure has been used widely in the past, but this has proved to be inadequate in explaining the complexities and idiosyncrasies of the personal experience of engaging in occupations. There have been many suggestions on how occupations could be better categorized experientially. For example, Wilcock, (1998), Rebeiro et al., (2001) and Hammel, (2004) discussed the dimensions of occupation as; doing, being, becoming and belonging. These concepts are 
widely used in occupational therapy literature but have been criticised for a lack of detailed description and justification (Hitch, Pepin and Stagnitti 2014 a \& b) leading to a knowledge practice gap. Pierce (2001a) suggested that occupations could be categorised as providing pleasure, productivity and restoration and Hammel (2009) also proposed four experience based categories of occupation; restorative; fostering belonging; doing; and reflecting life continuity. Jonsson (2008) identified seven experience-based categories of occupation including engaging, social and relaxing, as well as time killing and these were subsumed into two patterns that could be linked to wellbeing. This is a useful step in linking meaningful occupations to health and wellbeing.

Although these theoretical propositions have been useful in progressing discussion within occupational science and occupational therapy, there is now a need to relate this theory to the body of empirical research that has gathered around the subject of the personal meaning of engagement in an occupation (Hocking 2009). To date there has not been a synthesis of this research and it is unclear whether there are common dimensions of personal meaning attached to engagement in these different occupations. Thus, this study sought to systematically review the literature and distil similarities of personal meaning between autotelic occupations. The research question was: What meaning is attributed by adults to their engagement in autotelic occupations?

\section{Method}

\section{Study Design}

As a body of extant research existed, a systematic review of the literature was conducted to provide a synthesis of the findings. Having confirmed that there was no preexisting published systematic review, or one underway, the first stage of this metasynthesis was a scoping search. This led to refinement of the research terms and confirmed that studies on personal meaning were necessarily qualitative, focussing on individuals' lived experience of the phenomena. 
Qualitative metasynthesis refers to a set of techniques for the integration of qualitative findings (Centre for Reviews and Dissemination, (CRD)(2009). Ultimately the purpose of a metasynthesis is to bring together current research and propose a new interpretation of this knowledge for conceptual and theoretical development (Campbell, et al., 2011). The Joanna Briggs Institute, (JBI)(2014) identifies two main approaches to meta-synthesis: meta ethnography (which seeks new meaning) and meta aggregation (which does not seek to reinterpret). Meta aggregation was used in this study so that the essence of the findings of the primary studies was maintained. The study is reported in line with the Preferred Reporting Items for Systematic Reviews and Meta-Analyses (PRISMA) guidelines, (2016) (see Figure $1)$.

\section{Data Collection}

A systematic search of the literature was conducted during 2015-2016, using the electronic databases CINAHL, Medline, PsycInfo, SocIndex, and AMED and no date limit was imposed. Search terms were: Meaning* AND occupation OR activity AND "occupational science" AND interview. There is a potentially vast literature on meaning of occupations from across disciplines so the term "occupational science" was used to refine the findings by focussing on research from the same theoretical paradigm in the first instance. It is acknowledged that other disciplines have something to say about the meaning of occupations, but these were not sought as their purpose and theoretical stance are potentially dissimilar. For example, understanding of the term "occupation" is different outside of occupational science and occupational therapy. The term "interview" was used to find inductive research aimed at exploring personal meaning. The inclusion criteria were; empirical studies with the primary aim of investigating the meaning of an occupation from an individual's perspective; adults over the age of 18; papers in the English language in peer reviewed journals; and no date limit was applied. 
The initial database search conducted by the first reviewer yielded 87 papers. A further 13 papers were identified from other sources including hand search of the British Journal of Occupational Therapy and the Journal of Occupational Science. Eighty-one papers remained after duplicates were removed and the titles and abstracts of all these papers were screened by both authors and agreement reached on papers to exclude, without recourse to a third reviewer. Forty-four papers were excluded for various reasons, see Table 1 Thirty-seven full text articles were retrieved and read for eligibility by both authors. Seventeen were excluded for the reasons given in Table 2. Twenty studies remained following exclusions, see Figure 1.

(Tables 1\& 2)

(Figure 1).

Data were extracted from the papers and tabled (Table 3) by the first reviewer and checked by the second, under the headings of location, aim, methodology and analysis, themes and subthemes of the findings. The papers were then appraised by both reviewers independently, using the Rosalind Franklin Qualitative Research Appraisal Instrument (Henderson \& Rheault, 2004), which facilitates the scoring of papers according to the credibility, transferability, dependability and confirmability of the research process and findings (Table 3). Agreement was achieved without recourse to an additional reviewer. There were some common quality issues, some scoring at the lowest levels (4 and 5) (Table 4). However, no papers were excluded because the findings of these studies were coherent with studies that achieved a higher quality score. The location of the studies was international.

(Table 3)

(Table 4)

\section{Data Analysis}

The authors independently and subsequently in collaboration, conducted a process of meta-aggregation of the findings of the individual studies. The subthemes of the original 
studies were tabled (first order analyses). Then meta-aggregation of the data took place; the researchers grouped similar subthemes from the original studies together (second order analysis) (JBI, 2014), see Table 5. In an iterative process these subthemes were grouped and re-grouped visually several times in order to enable linking between them, before they were aggregated into the final overarching themes to produce a single comprehensive set of synthesized findings. The themes and subthemes are dynamically interconnected, so they are represented conceptually in Figure 2. Due to the interconnected nature of the themes there is no hierarchical significance to the order of the themes reported below.

(Table 5 here)

(Figure 2 here)

\section{Findings}

The aim of this study was to systematically review the literature to ascertain what meaning is attributed by adults to their engagement in autotelic occupations. Four themes of meaning were found and are described below: Occupations Provide a Sense of Fulfilment; Occupations Provide a Sense of Restoration; Occupations Shape Personal and Socio-cultural Identity and Occupations Provide Social, Cultural and Intergenerational Family Connection.

\section{Occupations Provide a Sense of Fulfilment}

The findings revealed that participants achieved a sense of fulfilment through engagement in the occupation as it provided opportunities for promoting their learning and development, enabling an experience of pride and satisfaction, and being challenged and achieving mastery as well as sometimes making contribution to others.

\section{Learning and personal development.}

Participants spoke about growing self-esteem or self-confidence from engagement in the occupation (Wright St Clair et al., 2004; Garci \& Mandich, 2005; Aguilar et al., 2010). 
Australian men and women computer users said that using the computer for leisure had raised their self-esteem and confidence, as they were able to demonstrate mastery and help others, which in turn led to acknowledgment of their skills by friends and family (Aguilar., et al 2010).

\section{Achieving mastery and establishing future goals.}

The review showed that occupations provided an opportunity for being challenged and achieving a goal, sometimes resulting in empowerment and a sense of control and sometimes awakening new goals and dreams for the future (Tzanidaki \& Reynolds 2011; Wensley \& Slade 2012). Skateboarders reported meeting the challenge and fulfilling personal goals as they mastered a new trick, which provided feedback that led them to seek out further challenges (Haines, Smith, \& Baxter 2010).

In some studies, the competitive element of the occupation was meaningful to individuals. For example, women recovering from breast cancer who competed in dragon boat racing, reported that wellbeing grew out of the physical achievement of competing; each person aiming for individual best (Unruh, \& Elvin, 2004). Similarly, older adult games players (tennis, bowling, dominos) reported self-challenge and personal accomplishment from the competitive element of their occupation (Hoppes, Wilcox, \& Graham, 2001).

\section{Pride and satisfaction.}

Closely linked to the challenge and achievement of engaging in an autotelic occupation was the sense of pride, satisfaction and accomplishment (Tzanidaki \& Reynolds, 2011; Roberts and Farrugia 2013). Participants in Pollanen's study (2013) reported pride in seeing their handicrafts in the homes of loved ones, which strengthened the sense of meaningfulness.

\section{Making a contribution.}

Studies in this review demonstrated that individuals reported meaning when their occupation made a contribution to the community or their family or friends (e.g. Shordike \& 
Pierce, 2005; Aguilar et al, 2010; Beagan \& D’Sylva, 2011; Sakuae \& Reid, 2012; Roberts \& Farrugia, 2013). In some studies where an end product was sold the participants reported being happy to contribute to the household financially (Boerema et al., 2010; Stephenson et al., 2013; Watters, Pearce, Backman, \& Suto, 2013). The wheelchair athletes in Garci and Mandich's (2005) study talked about the overwhelming honour of competing and Thai women engaged in preparing food for offering to the temple described a sense of intense fulfilment from honouring cultural norms and gaining merit in the next world (Wright St Clair et al., 2004).

\section{Occupations Provide a Sense of Restoration}

Most of the studies reviewed here reported that individuals experienced a sense of restoration from engaging in the occupation and this included happiness, emotional and spiritual feelings, an experience of stress relief, increased concentration and mental stimulation, sensory experiences, an enhanced sense of health and wellness, and being intensely in the moment. They also reported that they valued their occupation as it structured their day. There were some occasions when the occupation was not restorative, but instead created negative feelings.

\section{Enjoyment, pleasure and happiness.}

Participants described their engagement in an occupation as bringing about a sense of psychological wellbeing using terms such as happiness, relaxation, freedom, escape, peace and even ecstasy. This arose from a variety of occupations including active physical ones like recreational walking but also creative occupations like arts and crafts, cake decorating, sewing and singing. The immigrant women in Boerema and colleagues study (2010) said sewing made them feel happy and lifted their spirits.

A textile artist said that engagement in the occupation "gives me peace in my soul" (Tzanidaki and Reynolds 2011 p. 378). Similarly, cake decorators reported health benefits 
which included keeping the mind active and experiencing a sense of wellbeing and decreased stress (Sheerer et al., 2004). Choir singers reported feeling euphoric as a result of singing (Jacob et al., 2009) and the musicians in Roberts and Farrugia's (2013) study said that playing in a band "gets you in ecstasy" (p. 96). The older adult games players reported that playing keeps their mind off problems associated with daily life (Hoppes et al., 2001).

\section{Emotional, spiritual and sensory feelings.}

Sometimes participants reported a spiritual experience when engaging in an occupation and these feelings were closely related to feelings of happiness and fulfilment. One of the themes of meaning in Wensley and Slade's (2012) study of walkers was connection to nature, which gave them a sense of tranquillity. Choir singers reported that singing with the choir was almost spiritual (Jacob et al., 2009), leading to an experience of wholeness, feeling at ease with oneself and confirming of their sense of being. This heightened sense of spiritual or religious connection was also reported in the music making of Maltese band musicians (Roberts \& Farrugia, 2013) and the Cretan artists in Tzanidaki and Reynolds (2011) study, although the participants in both studies were recruited from religious groups which may have influenced the findings. Some participants valued the sensory aspects of their occupation, including sound, touch, colour, smell, taste, and sensory memories. For example, women noted that tea drinking was multisensory (Hannam, 1997).

\section{Relaxation and stress relief.}

Many of the studies in this review reported that occupations were relaxing, calming and soothing and offered participants stress relief. For example, craft makers said craft helped them during significant losses and unusually stressful times, such as illness, anorexia, depression, divorce, unemployment and illness or death of a loved one (Pollanen, 2013) and listening to or playing music was a way to relax, especially if one had felt angry about something (Eidevall \& Leufstadius, 2014). 


\section{Being intensely in the moment.}

Closely linked to the sense of happiness described above, the emotional sense of time passing in a deeply enjoyable way was reported in many of the reviewed studies. For example, cake decorators reported a meditative component to their engagement in the occupation and reported feelings of ecstasy and loss of awareness of their surroundings: "It's like zoning out in your own little world...I enjoy creating and once I start creating, truly creating, and my heart is into it, I just go into my own little world and I work till I'm finished" (Sheerer et al., 2004, p. 71).

\section{Sense of health and wellness and negative feelings.}

Some studies reported a sense of wellness and physical health from engagement in occupation (Unruh \& Elvin, 2004; Roberts \& Farrugia, 2013) including keeping the mind active (Sheerer et al., 2004). Occasionally there were negative feelings, for example when walkers experienced guilt over holding others back or frustration from being held back (Wensley and Slade, 2012).

\section{Structuring the day.}

Occupations were appreciated for the structure they bring to the day (Hoppes et al. 2001; Pollanen, 2013) and participants in Hannam's (1997) study felt tea drinking provided an excuse for a rest and was a way of dividing the day into manageable chunks.

\section{Occupations Shape Personal and Socio-cultural Identity.}

\section{Personal identity.}

The metasynthesis yielded four subthemes related to personal identity; self-perception and a sense of uniqueness; continuity of self; gendered occupations and occupations as part of daily life. Participants reported that the occupation was "always part of my life" (Boerema et al., p. 80), allowed them to be themselves (Eidevall \& Leufstadius, 2014), made them feel 
unique (Jacob et al., 2009), and reinforced their personal identity (Roberts \& Farrugia, 2013). According to Garci and Mandich (2005) the athletes were completely defined by their engagement; it became part of who they were and how they defined themselves.

Sometimes engagement in the occupation enhanced individual's identity. Women felt being a dragon boat racer formed their identity rather than the breast cancer (Unruh and Elvin, 2004). Occupations offered a chance to be recognised as a socially worthy individual, for example, an athlete or musician, rather than a person with a disability (Garci \& Mandich, 2005; Eidevall \& Leufstadius, 2014) or reinforced their identity, for example defining the self as artistic (Tzanidaki \& Reynolds, (2011). Similarly, Goan immigrant women felt that engaging in food related occupations maintained a gendered ethnic identity (Beagan \& D’Sylva, 2011) and Karen immigrant women reported weaving as an enduring aspect of their identity (Stephenson et al., 2013). Engagement in the occupation was an important part of their daily life (Aguilar et al., 2010), bound to their memories and life stories (Eidevall \& Leufstadius, 2014).

\section{Socio-cultural identity.}

The metasynthesis demonstrated how engagement in the occupation related to an individual's identity in relation to their family, social group and culture. Engagement in the occupation reinforced their identity as a family member (Beagan and D’Sylva, 2011), fulfilling their obligations to present and past relations (Wright St Clair et al., 2004). Some felt shaped by the engagement of past family members in the occupation (Boerema et al., 2010; Tzanidaki \& Reynolds, 2011), or by using objects handed down by family members (Hannam, 1997; Shordike \& Pierce, 2005). Others felt they were shaping future generations of their family, for example by teaching daughters to weave (Stephenson et al., 2013) or leaving an heirloom (Tzanidaki \& Reynolds, 2011).

Some studies in the review mentioned that group membership conferred a social identity and sense of belonging (Haines et al., 2010; Wensley \& Slade, 2012) and that this is 
intertwined with personal and cultural identity (Wright St Clair et al., 2004). Studies of preparing and cooking food, and weaving showed that the occupation reinforced a cultural identity, even when those involved had left their homeland (Beagan and D'Sylva, 2011; Stephenson et al., 2013). This subtheme is closely connected with personal identity described above and with cultural connection described in the next theme.

\section{Occupations Provide Social, Cultural and Intergenerational Family Connection.}

The metasynthesis yielded three subthemes of meaning in relation to connection with others; social/community support; connection to one's culture and intergenerational family connection.

\section{Social/community support.}

Social support derived from engagement in the occupation was a strong theme; occupation connected people to other people and their community. People valued the opportunity to develop friendships with those with similar interests, enjoying sharing recipe variations (Shordike \& Pierce, 2005), end products like food or tea (Hannam, 1997; Beagan \& D’Sylva, 2011) or art (Watters et al., 2013). Some people reported that engaging in their occupation as a team provided then with a sense of belonging (Hoppes et al. 2001; Garci \& Mandich, 2005). Weavers reported how they valued working together (Stephenson et al., (2013) and others reported developing a bond (Hannam, 1997; Jacob et al., 2009; Tzanidaki \& Reynolds, 2011).

A strong sub theme was the sense of support derived from connection with other people (Sheerer et al., 2004 ; Unruh \& Elvin, 2004; Sakuae \& Reid, 2012; Wensley \& Slade 2012; Roberts \& Farrugia, 2013; Stephenson et al., 2013). For example, participants recovering from breast cancer reported that they felt a shared understanding, and they experienced transcendence over the cancer experience through dragon boat racing, expressing: “oneness with all women who struggled against breast cancer" (Unruh and Elvin 2004 p. 144). 
The sense of support and connection to friends and groups of people contributed to a strong sense of belonging to a community (Wensley \& Slade, 2012; Roberts \& Farrugia, 2013). This included valuing hospitality and caring for others (Beagan \& D'Sylva, 2011; Sakuae \& Reid, 2012 ) and a sense of solidarity with others (Haines et al., 2010; Eidevall \& Leufstadius, 2014).

\section{Connection to one's culture.}

Studies in this review showed that occupations enabled people to enact rituals and experience cultural changes and transmission. In the studies describing food preparation and tea drinking the ritual of enacting the occupation was meaningful (Hannam, 1997; Wright St Clair et al., 2004; Shordike \& Pierce, 2005; Sakuae \& Reid 2012). In some studies, participants reported that the occupations were changing their form as customs changed over time and this incurred a sense of loss (Hannam, 1997; Shordike \& Pierce, 2005; Beagan and D'Sylva, 2011). People reported that engagement in the occupation held meaning because it facilitated a connection with their culture, preserving and transmitting it by passing on knowledge and skills (Scheerer et al., 2004; Beagan \& D’Sylva, 2011; Roberts \& Farrugia, 2013) and connecting with historical traditions (Boerema et al., 2010; Watters et al., 2013; Stephenson et al., 2013).

\section{Intergenerational family connection.}

There was a strong sense amongst the study participants that occupations connected them to family in the present, past and future. Engagement in the occupation linked them to memories of childhood and past family events (Hannam, 1997; Sheerer et al., 2004; Shordike \& Pierce, 2005; Eidevall \& Leufstadius, 2014). Sometimes it was objects that held memories connecting them to their family history across time (Hannam, 1997; Shordike \& Pierce, 2005).

Engaging in the occupation brought some people closer to deceased relatives (Sheerer et al., 2004; Roberts \& Farrugia, 2013; Eidevall \& Leufstadius, 2014). Elderly 
Greek women felt connected with earlier generations through engaging in the same occupations and leaving a legacy:

My grandmother, I remember that she made a painting and we kept it in our family as a precious heirloom. This piece is going to stay forever. ....You produce something and you leave it behind, (Tzanidaki \& Reynolds 2011 p. 379).

Some felt connected to their descendants by teaching them an occupation (Shordike \& Pierce, 2005; Stephenson et al., 2013; Watters et al., 2013). In Wright St Clair's study (2004) about preparation and serving traditional foods at Songkran, women expressed a strong sense of duty to continue the traditional methods, teaching children and grandchildren how this occupation had been done by their parents and grandparents.

\section{Discussion}

The purpose of this metasynthesis was to understand what meaning is attributed by adults to their engagement in an autotelic occupation and this has been achieved through a synthesis of existing empirical research. Synthesis of a variety of different occupations shows that occupations can provide a sense of restoration; a sense of fulfilment; social, cultural and intergenerational connection and shape personal identity. It is evident that these dimensions of meaning are dynamically interlinked (as depicted in figure 2).

Returning to the theoretical propositions outlined in the literature review, it is possible to see some synergy between the experiential concepts of doing, being, becoming and belonging (Wilcock, 1998, Rebeiro et al., 2001 and Hammel, 2004) and the findings of this metasynthesis; being with aspects of the theme of restoration, becoming with the theme of personal and social identity and belonging with the theme of social, cultural and intergenerational connection. The synergy between aspects of Pierce's (2001a) dimensions of pleasure, productivity and restoration and Hammel's (2009) experience based categories of occupation; restorative; fostering belonging; doing; and reflecting life continuity and the four 
themes of this metasynthesis can be observed, although Pierce (2001a) does not include social and cultural connection.

This metasynthesis has shown that engagement in autotelic occupations provides people with a sense of restoration. Joyful and spiritual emotions, complete absorption, relaxation and being intensely in the moment were all reported. This appears similar but not synonymous with the phenomenon of "flow" (Csikszentmihalyi, 1992). Czikszentmihalyi considered flow to include a balance between the challenge of the occupation and the skills of the individual. However, Ikuigu, Hoyme \& Mueller et al 2015 argued that flow inducing occupations (termed as psychologically rewarding occupations) were different to meaningful occupations, noting that psychologically rewarding occupations were always meaningful but the converse could not always be said. It would be premature to label the restorative effects of meaningful occupation seen in this review as flow and this issue requires further investigation and clarification. There has been interesting discussion within occupational science on how these kinds of restorative psychological experiences are conceptualised and potentially distinguished from flow (Wright, Sadlo, \& Stew, 2006, 2007; Ikuigu, Hoyme \& Mueller 2015) and mindfulness (Elliot, 2011). In a phenomenological study of seven people's flow experience Wright, Sadlo, \& Stew (2007) concluded that there were four different psychological experiences when people described the process of flow: challenge-skill, enjoyment, positive distraction and mindfulness. The similarities between these concepts and the subthemes in this metasynthesis are evident. However, in this work the words "being in the moment" were used to summarise the experience of being intensely absorbed in the current moment of occupational engagement, rather than adopting the popular term "mindfulness". There has been a tendency within occupational therapy literature to adopt terms from other disciplines in an uncritical way, without giving thought to carefully isolating and defining concepts as observed by occupational therapists and occupational scientists. The discussions around flow demonstrates that it is a complex concept and it is unclear if all the characteristics of flow as defined by Czikszentmihalyi (1992) need to be present for it to be 
named flow, or indeed if there are different psychological states that are restorative without the goal-challenge-skill element.

There is no doubt from the findings of this review that engagement in occupations had restorative benefits to individuals and this was core knowledge at the birth of occupational therapy. However, the benefits of restorative occupations to health and wellbeing deserves renewed attention now, as they have largely fallen from contemporary occupational therapy literature. Indeed in 2000 they were referred to as the "forgotten dimension" (Howell and Pierce 2000) and this is still largely true today. As occupations that relax and enhance a sense of health and wellbeing are increasingly a focus of health promotion, it is time for occupational therapists to refocus their skills in offering individual's engagement in occupations that are personally meaningful to provide restorative feelings and thus enhance happiness, health and feelings of wellbeing.

The findings of this metasynthesis demonstrate clearly that there is an empirical basis to the theoretical propositions that engagement in occupations can bring about a sense of social connection and social support from friends and the community and a rich intergenerational connection to family and culture. Furthermore, this positive experience of connection contributes towards making the chosen occupation meaningful and thus potentially health enhancing. Hammel (2014) proposed that occupational therapy theory should embrace occupations fostering a sense of belonging, specifically doing things with and for others (Hammel 2014). According to Hammel (2014) meaning is attributed to and derived from occupations that foster connections to ancestors and offer opportunities for social collaboration and strengthening of social roles. Several empirically derived themes from this review support this, (making a contribution to community and others; the value of social/community support; connection to one's culture and intergenerational family connection). 
Furthermore, one of the dimensions of personal meaning derived from engagement in an autotelic occupation found in this review was shaping individual's sociocultural identity, which is clearly linked to Hammel's (2014) sense of social belonging. This metasynthesis concurs that socio-cultural connection and shaping of social identity are meaningful aspects of an individual's autotelic occupations. The importance of engaging in meaningful occupations to shape, maintain and change an individuals' identity is well understood in occupational therapy and occupational science (Christiansen and Townsend 2004; Taylor and Kay 2013) and this review provides further empirical support and rationale for occupational therapy.

This metasynthesis indicates that when people engage in an autotelic occupation, regardless of the form of the occupation, that there are some similarities in terms of the dimensions of personal meaning that are derived. These findings help to advance the theoretical understanding of the meaning of people's autotelic occupations, so that this knowledge can be linked to other bodies of knowledge, in what Pierce (2012) described as relational research. This knowledge can be used to understand and articulate the therapeutic value of meaningful occupations for health promotion, and the reasons for sustained engagement in healthy occupations. Ultimately this facilitates its application in a prescriptive way (Pierce, 2012) in the applied sciences, for example, occupational therapy.

\section{Study Limitations}

The search was wide ranging and rigorous, but hand searching could have been more extensive, and it is possible this may have yielded further papers. Similarly, the term occupational science was used as a search term rather than occupational therapy; this was a deliberate decision to limit the literature to primary studies of meaning rather than therapeutic benefit. At this point in time, a review needed to synthesise the descriptive body of knowledge (present predominantly in the OS literature), rather than jumping ahead to prescriptive literature. Despite this potential limitation, studies from four Occupational Therapy journals arose in the search and were included in the review. The findings of this 
review can only reflect the quality of the studies included and it was noted above that the quality of the studies was often poor. However, despite the weak quality, the similarity between findings enhances their credibility through triangulation. Furthermore, although there were studies from eastern and western cultures, they were all published in English, so it is unknown whether studies exist in other languages that could enrich the findings. There was also a predominance of female participants in the studies. Finally, the strong presence of cultural issues in the findings of this review may have been influenced by the presence of several studies conducted on immigrant women who were experiencing occupational alienation (Boerema et al., 2010; Beagan \& D’Sylva, 2011; Stephenson et al., 2013).

\section{Implications for Practice}

The dimensions of personal meaning arising from engagement in an occupation make it clear why occupations can have therapeutic value. An autotelic occupation can provide fulfilment, restoration, enhanced personal identity, and connection with fellow humans now and over time. These findings demonstrate the power of engagement in an occupation to sustain and restore health and wellbeing and reinforce the enormous potential of occupational therapists to use occupation prescriptively to promote health and wellbeing.

Occupational therapists have long used occupations to help clients to set and meet challenges and to experience the resulting sense of fulfilment. This metasynthesis provides some empirical support to the notion that occupations can bring about a sense of fulfilment in people. Occupational therapists have the skills to enable just the right amount of challenge to facilitate this sense of fulfilment (the "just right challenge" Yerxa, 1990). This skill is currently important in the field of public health, where increasing emphasis is being placed on using occupations to maintain or restore health. Furthermore, the role of occupations in shaping personal identity has long been acknowledged in occupational therapy. It is understood that identity is related to what people do and it is central to coherence, meaning and wellbeing as our life story unfolds (Christiansen 1999). Humans express themselves 
through occupation and understand more about themselves through being engaged in occupation (Hasselkus 2011). The findings of this metasynthesis confirm that occupations are meaningful in relation to personal and social identity and this provides occupational therapists with further justification for using occupations therapeutically.

The importance of occupations in providing social, cultural and intergenerational connection for people is very clear in these findings, as evidenced by studies from many different cultures. This has potential therapeutic value for occupational therapists, to reconnect people to their families, local communities and society, enhancing their sense of belonging and ultimately their health and wellbeing, as intimated by Wilcock (2006) and Hammel (2014). This also has important implications for public health professionals seeking to engage individuals in meaningful occupations for the benefit of their health.

\section{Further Research}

It would be interesting to know if these dimensions of meaning relate to studies not reported in the English language and to occupations that are not autotelic. It would also be interesting to examine whether the dimensions relate to a wider range of autotelic occupations Translation of the findings of this synthesis into the bodies of work of other disciplines will be the next necessary stage of knowledge development.

\section{Conclusion}

This metasynthesis addressed the question of what meanings people attach to their engagement in autotelic occupations. The study found four main themes of personal meaning. Engagement in occupations have the potential to connect individuals to a community and culture and this social, cultural and intergenerational connection enhances and shapes personal and social identity. Furthermore, meaning was derived from opportunities for fulfilment and personal enrichment, as well as restorative experiences and these experiences are clearly linked to individuals' feelings of health and wellbeing. These findings have some 
synergy with current theoretical propositions but provide further impetus to refining the dimensions of meaning derived for engagement in autotelic occupations. They also provide occupational therapists with a strong empirical rationale for using meaningful occupations to enhance individuals' sense of health and wellbeing.

\section{Key Messages}

- This synthesis of empirical findings advances theory of the dimensions of meaning of autotelic occupations to individuals: There is a clear link between engagement in a personally meaningful autotelic occupation and an individual's sense of personal and social wellbeing

- Four common dimensions of personal meaning identified in this synthesis of empirical research findings provide occupational therapists with an evidence base for the therapeutic value of engagement in personally meaningful occupations

\section{References}

*Aguilar, A., Boerema, C., \& Harrison, J. (2010). Meanings attributed by older adults to computer use. Journal of Occupational Science, 17, 27-33.

doi:10.1080.14427591.2010.9686669

*Beagan, B. L., \&D’Sylva, A. (2011). Occupational meanings of food preparation for Goan Canadian women. Journal of Occupational Science, 18, 210-222. doi: $10.1080 / 14427591.586326$

*Boerema, C., Russell, M., \& Aguilar, A. (2010) Sewing in the lives of immigrant women. Journal of Occupational Science, 17, 78-84. doi:10.80/14427591.2010.9686678 
Campbell, R., Pound, P., Morgan, M., Daker-White, G., Britten, N., Pill, R., Donovan, J. (2011). Evaluating meta-ethnography: systematic analysis and synthesis of qualitative research. Health Technology Assessment, 15, 43. doi: 10.3310/hta15430

Centre for Reviews and Dissemination (2009). Systematic Reviews. CRD's guidance for undertaking reviews in health care. CRD: University of York. https://www.york.ac.uk/media/crd/Systematic_Reviews.pdf

Christiansen, C. (1999). Definite Lives: Occupation as identity: an essay on competence, coherence and the creation of meaning. American Journal of Occupational Therapy, 53, 547- 56. doi:10.5014/ajot.53.6.547

Christiansen, C. (2004) Occupation and identity: Becoming who we are through what we do. In Christiansen C. and Townsend E. A. (2004) Introduction to Occupation. New Jersey; Prentice Hall.

Csikszentmihalyi, M. (1992). Flow. The psychology of happiness. London, UK: Rider.

*Eidevall, K., \& Leufstadius, C. (2014). Perceived meaningfulness of music and participation in a music group among young people with physical disabilities. Journal of Occupational Science, 21, 130-142. doi:10.1080/14427591.2013.764817

Elliot, M. L. (2011). Being mindful about mindfulness: An invitation to extend occupational engagement into the growing mindfulness discourse. Journal of Occupational Science, 18, 366-376. doi: 10.1080/14427591.2011.610777

*Garci, T., \& Mandich, A. (2005). Going for Gold: Understanding elite level wheelchair basketball athletes. Journal of Occupational Science, 12, 170-175. doi:10.1080/14427591.2005.9686560 
*Haines, C., Smith, T. M. \& Baxter, M. F. (2010). Participation in risk-taking occupation of skateboarding. Journal of Occupational Science, 17, 239-245. doi: 10.1080 .14427591 .2010 .9686701

Hammell, K. W. (2004). Dimensions of meaning in the occupations of daily life. Canadian Journal of Occupational Therapy, 5, 296-305. doi: 10.1177/000841740407100509

Hammell, W. H. (2009a). Sacred texts: A sceptical exploration of the assumptions underpinning theories of occupation. Canadian Journal of Occupational Therapy, 76, 613. doi: $10.1177 / 000841740907600105$

Hammell, K. W. (2009b). Self-care, productivity, and leisure, or dimensions of occupational experience? Rethinking occupational "categories”. Canadian Journal of Occupational Therapy, 76, 107-113. doi: 10.1177/000841740907600208

*Hannam, D. (1997). More than a cup of tea: Meaning construction in an everyday occupation. Journal of Occupational Science Australia, 4, 69-74. doi:10.1080/14427591.1997.9686423

Hasselkus, B. R. (2011). The meaning of everyday occupation ( $2^{\text {nd }}$ ed.) Thorofare, New Jersey: Slack Incorporated.

Henderson, R., \& Rheault, W. (2004). Appraising and incorporating qualitative research in evidence-based practice. Journal of Physical Therapy Education, 18, 35-40. ISSN: 0899-1855 AN: 106612210

Hinojosa, J., \& Kramer, P. (1997). Statement: Fundamental concepts of occupational therapy: Occupation, purposeful activity and function. American Journal of Occupational Therapy, 51, 864-866. doi: 10.5014/ajot.51.10.864

Hitch, D., Pepin G., \& Stagnitti, K. (2014a) In the Footsteps of Wilcock, Part one: The evolution of doing, being, becoming and belonging. Occupational Therapy in Health Care, 28 231-246 doi: 10.3109/07380577.2014.898114 
Hitch, D., Pepin G., \& Stagnitti, K. (2014b) In the Footsteps of Wilcock, Part two: The interdependent nature of doing, being, becoming and belonging. Occupational Therapy in Health Care, 28 247-263 doi: 10.3109/07380577.2014.898115

*Hoppes, S., Wilcox, T., \& Graham, G. (2001). Meanings of play for older adults. Physical \& Occupational Therapy in Geriatrics, 18, 57-68. doi: 10.1080/J148v18n03 04

Ikuigu, M. N., Hoyme, A. K., Mueller, B. A., Rochelle, R. R. (2015). Meaningful occupation clarified: Thoughts about the relationship between meaningful and psychologically rewarding occupations. South African Journal of Occupational Therapy 45, 4750. doi.org/10.17159/2310-3833/2015/v45no1a8

*Jacob, C., Gupthill, C., \& Sumsion, T. ( 2009). Motivation for continuing involvement in a leisure based choir: The lived experiences of university choir members. Journal of Occupational Science, 16, 187-193. doi: 10.1080.14427591.2009.9686661

Joanna Briggs Institute (2014). Joanna Briggs Institute Reviewers' Manual. South Australia : University of Adelaide. http://joannabriggs.org/assets/docs/sumari/reviewersmanual$\underline{2014 . p d f}$

Jonsson, H. (2008) A new direction in the conceptualization and categorization of occupation, Journal of Occupational Science, 15:1, 3-8, DOI: 10.1080/14427591.2008.9686601

Pierce, D. (2012). The 2011 Ruth Zemke lecture in Occupational Science: Promise. Journal of Occupational Science, 19, 298-311. doi: 10.1080.14427591.2012.667778

Pierce, D. (2001). Occupation by design: Dimensions, therapeutic power and creative process American Journal of Occupational Therapy, 55, 249-259. doi: 10.5014/ajot.55.3.249

Polatajko, H. J., Davis, J., Stewart D., Cantin, N., Amorosos B., Purdie L., Zimmerman D. (2013a) Specifying the domain of concern: Occupation as core. (pp13-36) In Townsend, E. A., \& Polatajko, H. J. (Eds). Enabling Occupation 11: Advancing an occupational therapy vision for health, well-being and justice through occupation. $2^{\text {nd }}$ ed. Ottawa, ON: CAOT Publications ACE. ISBN 978-1-895437-89-8. 
Polatajko, H. J., Backman, C., Baptiste, S., Davis, J., Eftekhar, P., Harvey, A., Jarman, J., ... Connor-Schisler, A. (2013b) Human occupation in context (pp37-61). In Townsend, E. A., \& Polatajko, H. J. (Eds.) Enabling Occupation 11: Advancing an occupational therapy vision for health, well-being and justice through occupation. $2^{\text {nd }}$ ed. Ottawa, ON: CAOT Publications ACE. ISBN 978-1-895437-89-8.

*Pollanen, S. (2013). The meaning of craft: craft makers' descriptions of craft as an occupation. Scandinavian Journal of Occupational Therapy, 20, 217-227. doi: $10.3109 / 11038128.2012 .725182$

PRISMA, (2016). Retrieved from http://www.prisma-statement.org/Default.aspx

Rebeiro, K. L., Day, D., Semeniuk, B., O'Brien, M., and Wilson, B. (2001). Northern Initiative for Social Action: An occupation based mental health program. American Journal of Occupational Therapy, 55, 493-500. doi: 10.5014/ajot.55.5.493

*Roberts, A. E. K., \& Farrugia, M. D. (2013). The personal meaning of music making to Maltese band musicians. British Journal of Occupational Therapy, 76, 94-100. doi: $10.4276 / 030802213 X 13603244419275$

*Sakuae, M., \& Reid, D. (2012). Making tea in place. Journal of Occupational Science, 19, 283-291. doi: 10.1080/14427591.2011.610775

*Sheerer, C. R., Cahill, L. G., Kirby, K., \& Lane, J. (2004). Cake decorating as occupation: Meaning and motivation. Journal of Occupational Science, 11, 68-74. doi:10.1080/14427591.2004.9686533

*Shordike, A., \& Pierce, D. (2005). Cooking up Christmas in Kentucky: Occupation and tradition in the stream of time. Journal of Occupational Science, 12, 140-148. doi: $10.1080 / 14427591.2005 .9686557$ 
*Stephenson, S. M., Smith, Y. J., Gibson, M., \& Watson, V. (2013). Traditional weaving as an occupation of Karen refugee women. Journal of Occupational science, 20, 224-235. doi: $10.1080 / 14427591.2013 .789150$

Taylor, J, \& Kay, S. (2013). The construction of identities in narratives about serious leisure occupations. Journal of Occupational Science, 1-17 doi:10.1080/14427591.2013.803298

*Tzanidaki, D., \& Reynolds, F. (2011). Exploring the meanings of making traditional arts and crafts among older women in Crete, using interpretative phenomenological analysis. British Journal of Occupational Therapy, 74, 375-382. doi: $10.4276 / 030802211 X 13125646370852$

*Unruh, A. M., \& Elvin, N. (2004). In the eye of the dragon: Women's experience of breast cancer and the occupation of dragon boat racing. Canadian Journal of Occupational Therapy, 71, 138-149. doi: 10.1177/000841740407100304

*Watters, A. M., Pearce, C., Backman, C. L. \& Suto, M. J. (2013). Occupational engagement and meaning: The experience of ikebana practice. Journal of Occupational Science, 20, 262-277. doi: 10.1080/14427591.2012.709954

*Wensley, R. \& Slade, A. (2012). Walking as a meaningful leisure occupation: The implications for occupational therapy. British Journal of Occupational Therapy, 75, 8592. doi: $10.4276 / 030802212 X 13286281651117$

Wilcock, A. A. (1998). Reflections on doing, being and becoming. Canadian Journal of Occupational Therapy, 65, 248-256. doi: 10.1177/000841749806500501

Wilcock, A. A. (2006). An occupational perspective of health ( $2^{\text {nd }}$ ed.). Thorofare, NJ: Slack Inc.

World Federation of Occupational Therapists. (2016). Definition of occupation. Retrieved from 
http://www.wfot.org/aboutus/aboutoccupationaltherapy/definitionofoccupationaltherapy . $\operatorname{aspx}$

Wright, J., \& Sadlo, G., \& Stew, G. (2006). Challenge-skills and mindfulness: An exploration of the conundrum of flow process. Occupational Therapy Journal of Research:

Occupation, Participation and Health. 26. 25-32. Doi:10.1177/153944920602600104.

Wright, J., Sadlo, G., \& Stew, G. (2007). Further explorations into the conundrum of flow process. Journal of Occupational Science, 14, 136-144.

doi:10.1080.14427591.2007.9686594

*Wright St Clair, V., Wannipa, B., Vittayakorn, S., Rattakorn, P., \& Hocking, C. (2004). Offerings: Food traditions of older Thai women at Songkran. Journal of Occupational Science, 11, 115-124. doi:10.1080.14427591.2004.9686539

Yerxa, E. J., Clark, F., Frank, G., Jackson, J., Parham, D., Pierce, D., Stein, C. \& Zemke, R. (1990). An introduction to occupational science. A foundation for occupational therapy in the 21st century. Occupational Therapy in Health Care, 6, 1-17. doi: 10.1080/J003v06n04_04 